Toward transferable interatomic van der Waals interactions without electrons: The role of multipole electrostatics and many-body dispersion

Tristan Bereau' and O. Anatole von Lilienfeld

Citation: The Journal of Chemical Physics 141, 034101 (2014); doi: 10.1063/1.4885339

View online: http://dx.doi.org/10.1063/1.4885339

View Table of Contents: http://aip.scitation.org/toc/jcp/141/3

Published by the American Institute of Physics

Articles you may be interested in

Blind test of density-functional-based methods on intermolecular interaction energies

The Journal of Chemical Physics 145, 124105124105 (2016); 10.1063/1.4961095

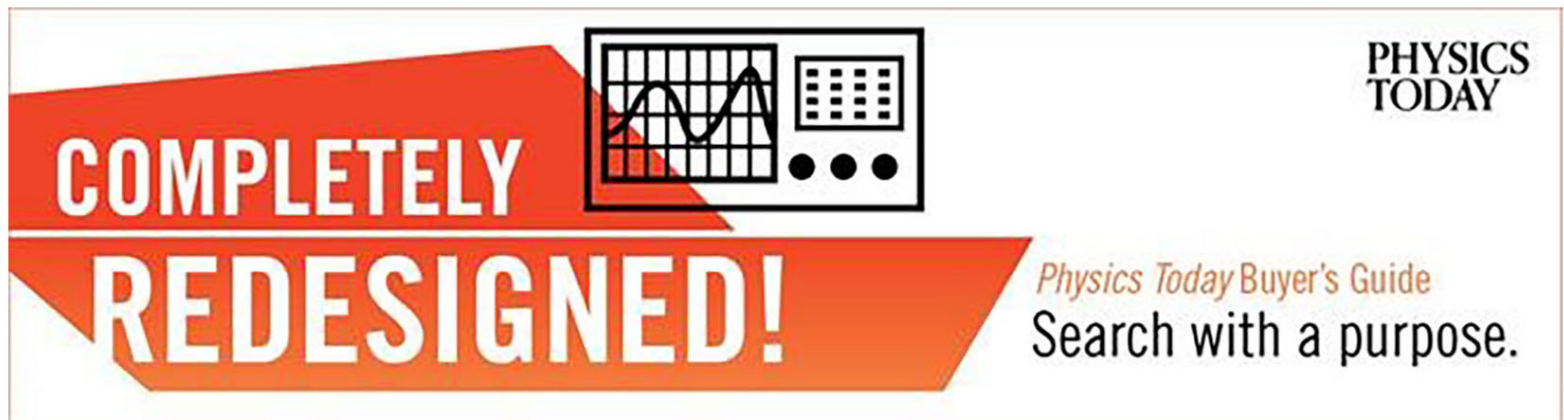




\title{
Toward transferable interatomic van der Waals interactions without electrons: The role of multipole electrostatics and many-body dispersion
}

\author{
Tristan Bereau ${ }^{1, a)}$ and O. Anatole von Lilienfeld ${ }^{2}$ \\ ${ }^{1}$ Max-Planck-Institut für Polymerforschung, Ackermannweg 10, 55128 Mainz, Germany and \\ Department of Chemistry, University of Basel, 4056 Basel, Switzerland \\ ${ }^{2}$ Department of Chemistry, Institute of Physical Chemistry, University of Basel, 4056 Basel, Switzerland and \\ Argonne Leadership Computing Facility, Argonne National Laboratory, Argonne, Illinois 60439, USA
}

(Received 21 March 2014; accepted 12 June 2014; published online 15 July 2014)

\begin{abstract}
We estimate polarizabilities of atoms in molecules without electron density, using a Voronoi tesselation approach instead of conventional density partitioning schemes. The resulting atomic dispersion coefficients are calculated, as well as many-body dispersion effects on intermolecular potential energies. We also estimate contributions from multipole electrostatics and compare them to dispersion. We assess the performance of the resulting intermolecular interaction model from dispersion and electrostatics for more than 1300 neutral and charged, small organic molecular dimers. Applications to water clusters, the benzene crystal, the anti-cancer drug ellipticine-intercalated between two Watson-Crick DNA base pairs, as well as six macro-molecular host-guest complexes highlight the potential of this method and help to identify points of future improvement. The mean absolute error made by the combination of static electrostatics with many-body dispersion reduces at larger distances, while it plateaus for two-body dispersion, in conflict with the common assumption that the simple $1 / R^{6}$ correction will yield proper dissociative tails. Overall, the method achieves an accuracy well within conventional molecular force fields while exhibiting a simple parametrization protocol. (C) 2014 AIP Publishing LLC. [http://dx.doi.org/10.1063/1.4885339]
\end{abstract}

\section{INTRODUCTION}

Predicting potential energies from first principles is a long-standing endeavor in physical chemistry. Post-HartreeFock methods offer a great deal of accuracy and transferability by solving Schrödinger's equation, though at the expense of unfavorable scaling as a function of system size. ${ }^{1}$ Density functional theory (DFT), on the other hand, implies only cubic scaling through the use of electron density and singleparticle orbitals alone. ${ }^{2}$ DFT has proven tremendously useful for the simulation of chemical systems and even for obtaining thermodynamical properties through the use of $a b$ initio molecular dynamics. ${ }^{3,4}$ Unfortunately, common approximations in the employed exchange-correlation potential can result in significant shortcomings, such as the inadequateoccasionally even qualitatively wrong-predictions of the intermolecular forces that arise due to noncovalent vander-Waals (vdW) binding, ${ }^{5}$ or a dramatic overestimation of molecular polarizabilities. ${ }^{6}$ While the latter can usually be cured through the use of hybrid density functionals, ${ }^{7}$ the issue of vdW binding has motivated the development of a wide variety of dispersion-corrected methods, ${ }^{8-10}$ many of which offer an atom-pairwise additive ad hoc correction with the correct dissociative polynomial behavior of London's dispersion formula, $C_{6} / R^{6}$, referred to in the following as twobody dispersion (TBD). ${ }^{11-13}$ Even though the addition of such atom pairwise corrections very much improve the accuracy of DFT, ${ }^{14}$ it has also been demonstrated that the interatomic many-body vdW forces are not properly accounted for within LDA, GGAs, meta-GGAs, and hybrid functionals. ${ }^{15}$ Using

\footnotetext{
a)Electronic mail: bereau@mpip-mainz.mpg.de
}

the Axilrod-Teller-Muto expression, the magnitude of interatomic three-body dispersion forces has subsequently been found to be quite significant in many systems, particularly when going beyond isolated molecular dimers toward larger molecular assemblies or condensed-phase systems such as molecular liquids or crystals. ${ }^{16}$ To properly account for manybody effects, ${ }^{17,18}$ a many-body dispersion (MBD) method has recently been introduced ${ }^{19}$ that builds on the TkatchenkoScheffler (TS) ${ }^{20}$ TBD. Within MBD, free-atom polarizabilities are coupled at short interatomic distances by means of partitioning the atom around its closest neighbors according to its electron density. The MBD energy up to infinite order (i.e., $R^{-n}, n=\{6,9, \ldots\}$ ) is then obtained by diagonalizing the Hamiltonian corresponding to a system of coupled fluctuating harmonic dipoles ${ }^{21}$ thereby coupling the polarizabilities at long range as well. The importance of MBD has been demonstrated for a large variety of molecular assemblies and systems. ${ }^{19,22-24}$

In this work, we probe the ability to represent dispersion interactions using the TBD and MBD methods without an underlying electron density. Though initially developed to correct DFT calculations, the model provides a sound and efficient way to compute two-body and many-body dispersion with a minimal amount of free parameters and assumptions. This strategy follows a number of extensive efforts toward modeling largely classical potentials derived from rigorous quantum-mechanics, such as SIBFA ${ }^{25,26}$ and EFP, ${ }^{27}$ that partition the interaction energy into specific terms, e.g., static electrostatics, polarization, repulsion, dispersion, and charge transfer. As a promising compromise, the AMOEBA force field ${ }^{28}$ provides a number of refinements beyond 
standard atomistic models (e.g., multipole (MTP) electrostatics, polarization) that leads the way toward next-generation force fields. ${ }^{29}$ Far from competing with these established methods, the present work explores the prospects of an "electron-free" yet physics-based approach to dispersion interactions. The present work only considers dispersion alongside static multipole electrostatics, ${ }^{30}$ and thus lacks critical terms toward a complete intermolecular potential (more below). To the best of our knowledge, such a systematic model of MBD interactions has not yet been applied to compute intermolecular energies without any underlying electron density - all abovementioned force fields and potentials rely instead on pairwise interactions. Alternatively, the TBD approach we use here, though based on a standard $R^{-6}$ functional form, adapts the associated $C_{6}$ coefficients to the local chemistry (e.g., hybridization) and geometry of the system. The common usage of $R^{-6}$ functions and the present TBD method's limited computational overhead makes it relevant for force-field-based molecular modeling.

In Sec. II, we introduce the approximations and changes necessary to compute MBD and TBD energies without electron density, i.e., from Voronoi partitioning only. Details for calculating the MTP electrostatics are also provided. Next, we optimize the free parameters to best reproduce numbers for a training set of experimental molecular polarizabilities, as well as a small set of intermolecular energies of gas-phase dimers (Sec. IV). We then report the method's performance for a large variety of systems: Sec. III reports on intermolecular energies for a diverse set of 1000 hydrogen-bonded and dispersion-bonded complexes, neutral and charged aminoacid side chains, ionic groups, organic halides, halohydrides, and halogen molecules. Thereafter, we assess the method in more detail for a select set of interesting systems. Specifically, we discuss potential energies of binding for the water dimer, 38 water clusters with 2-10 monomers, the benzene crystal, and seven supramolecular host-guest complexes including the aromatic ellipticine drug and DNA base-pair dimer complex.

\section{METHODS}

\section{A. Effective atomic polarizabilities}

The frequency-dependent polarizability of atom $p$ can be expressed as a truncated Padé series,

$$
\alpha_{p}(i \omega) \approx \frac{\alpha_{p}^{0}[n(\mathbf{r})]}{1+\left\{\omega / \omega_{p}[n(\mathbf{r})]\right\}^{2}}
$$

where $\alpha_{p}^{0}[n(\mathbf{r})]$ is the static polarizability, $\omega_{p}[n(\mathbf{r})]$ is the corresponding characteristic excitation frequency, and $n(\mathbf{r})$ is the electron density. The two quantities $\alpha_{p}$ and $\omega_{p}$ in Eq. (1) are linked to the leading pairwise dispersion coefficient term $C_{6}$ for atoms $p$ and $q$ via the Casimir-Polder integral, ${ }^{31}$

$$
C_{6 p q}=\frac{3}{\pi} \int_{0}^{\infty} \mathrm{d} \omega \alpha_{p}(i \omega) \alpha_{q}(i \omega)
$$

For $p=q$, this yields $\omega_{p}=4 C_{6 p p} / 3\left(\alpha_{p}^{0}\right)^{2}$. $\mathrm{TS}^{20}$ proposed to compute $\alpha_{p}^{0}$ of an atom in a molecule via the ratio of its effective and free volumes, $\alpha_{p}^{0}=\alpha_{p}^{\text {free }}\left(V_{p}^{\text {eff }} / V_{p}^{\text {free }}\right)$, as obtained through Hirshfeld partitioning of electron densities,

$$
\frac{V_{p}^{\mathrm{eff}}}{V_{p}^{\text {free }}}=\frac{\int \mathrm{d} \mathbf{r} r^{3} w_{p}(\mathbf{r}) n(\mathbf{r})}{\int \mathrm{d} \mathbf{r} r^{3} n_{p}^{\text {free }}(\mathbf{r})}
$$

Here, $n_{p}^{\text {free }}(\mathbf{r})$ is the electron density of the free atom $p$ and $w_{p}(\mathbf{r})$ weighs the contribution of the free atom $p$ with respect to all free atoms at position $\mathbf{r}^{20}$ Accurate free-atom reference values of $\alpha_{p}$ and $C_{6 p p}$ can be found in a study from Chu and Dalgarno. ${ }^{32}$

In this work, we relax the requirement for an electron density $n(\mathbf{r})$. Instead, the free-atom density is represented by a Gaussian function centered at the nucleus, and the free-atom radius, $R_{p}^{\mathrm{vdW}},{ }^{16}$ is used as its width. We also replace the total electron density in the numerator by this free-atom density. As a proxy for the weight $w_{p}(\mathbf{r})$, we partition space into two regions,

$$
w_{p}(\mathbf{r}) \approx\left\{\begin{array}{lc}
1, & \mathbf{r} \in \mathcal{R}_{p} \\
\exp \left(-d\left(\mathbf{r}, \mathbf{r}_{p}\right) /\left(d_{w} R_{p}^{\mathrm{vdW}}\right)\right), & \text { otherwise }
\end{array}\right.
$$

where $\mathbf{r}_{p}$ is the position of atom $p, d\left(\mathbf{r}, \mathbf{r}_{p}\right)=\left|\mathbf{r}-\mathbf{r}_{p}\right|^{2}$ is the Euclidean distance between points $\mathbf{r}$ and $\mathbf{r}_{p}$, and $d_{w}$ is an atom-type independent, free parameter that scales the exponential decay. The partitioning between the region belonging to atom $p, \mathcal{R}_{p}$, and the rest relies on a Voronoi-type tessellation scheme ${ }^{33}$ to determine which atom is closest to any position $\mathbf{r}$,

$$
\mathcal{R}_{p}=\left\{\mathbf{r} \in \mathbb{R}^{3} \mid d\left(\mathbf{r}, \mathbf{r}_{p}\right) \leq d\left(\mathbf{r}, \mathbf{r}_{j}\right) \text { for all } j \neq p\right\} .
$$

In practice, $\mathcal{R}_{p}$ is evaluated from a set of points $\mathbf{r}$ located around atom $p$. While the Voronoi scheme systematically aims at the midplane between two atoms, the asymmetry between different chemical elements is encoded in $w_{p}(\mathbf{r})$ via the free-atom radii $R_{p}^{\mathrm{vdW}}$. To evaluate Eq. (3), we perform a discrete integration over a cubic grid of size $20 \mathrm{Bohrs}^{3}$ with step sizes of 1.0 Bohr around each atom $p$.

We further note that this partitioning does not allow to distinguish energetic differences between different charged states of a molecule from dispersion. Any effect due to a net charge is hereby only imprinted in the static electrostatics.

\section{B. MBD energy}

Originally, the MBD scheme prescribed a prior longrange coupling of the atomic polarizabilities. ${ }^{19}$ We note that more recently, it has been shown that the coupled quantum harmonic oscillator (QHO) model already includes longrange electrodynamic screening. ${ }^{34}$ In that procedure, shortranged screening effects are excluded from the MBD equation to avoid double counting of the DFT correlation energy and the polarizability screening. We hereby omit this separation, since our scheme does not entail any correlation, and work instead with the full interaction tensor.

The MBD energy is computed from the coupled fluctuating dipole model for a collection of coupled isotropic QHOs, each representing an atom in the system. ${ }^{19,21}$ We rely on the abovementioned set of effective atomic polarizabilities, $\alpha_{p}^{0}$, and their corresponding characteristic frequencies, $\omega_{p}^{0}$. The 
energy is obtained by diagonalizing the $3 N \times 3 N$ matrix

$$
C_{p q}^{\mathrm{QHO}}=\left(\omega_{p}\right)^{2} \delta_{p q}+\left(1-\delta_{p q}\right) \omega_{p} \omega_{q} \sqrt{\alpha_{p} \alpha_{q}} \mathcal{T}_{p q},
$$

where $\mathcal{T}_{p q}=\nabla_{\mathbf{r}_{p}} \otimes \nabla_{\mathbf{r}_{q}} W\left(r_{p q}\right)$ is the dipole interaction tensor, $\mathbf{r}_{p}$ and $\mathbf{r}_{q}$ are the atoms' positions, and $\delta$ is Kronecker's delta. Here, the dipole interaction tensor relies on the modified Coulomb potential

$$
W\left(r_{p q}\right)=\frac{1-\exp \left[-\left(\frac{r_{p q}}{R_{p q}^{\text {vdW }}}\right)^{\beta}\right]}{r_{p q}},
$$

where $r_{p q}=\left|\mathbf{r}_{p}-\mathbf{r}_{q}\right|, \beta$ is a range-separation parameter and $R_{p q}^{\mathrm{vdW}}$ is proportional to the sum of the vdW radii for a pair of atoms $p$ and $q: R_{p q}^{\mathrm{vdW}}=\gamma_{\mathrm{MBD}}\left(R_{p}^{\mathrm{vdW}}+R_{q}^{\mathrm{vdW}}\right)$, where $\gamma_{\mathrm{MBD}}$ is an atom-type independent, free parameter. The $\mathrm{vdW}$ radius of an atom is scaled according to the Tkatchenko-Scheffler scheme, $R_{p}^{\mathrm{vdW}}=\left(\alpha_{p} / \alpha_{p}^{\text {free }}\right)^{1 / 3} R_{p}^{\mathrm{vdW} \text {,free }}$. The free-atom vdW radii are formally obtained by measuring electron density contours for rare-gas atoms and extending the result to other atoms of the same row. ${ }^{20}$ All vdW radii used in the present work are reported in Ref. 16, except for iodine, which we determined as 4.39 Bohrs. The modified dipole interaction tensor for Cartesian components $a$ and $b$ between atoms $p$ and $q$ yields

$$
\begin{aligned}
\mathcal{T}_{p q}^{\prime a b}= & -\frac{3 r_{p q}^{a} r_{p q}^{b}-r_{p q}^{2} \delta_{a b}}{r_{p q}^{5}}\left(1-\exp \left[-\left(\frac{r_{p q}}{R_{p q}^{\mathrm{vdW}}}\right)^{\beta}\right]\right. \\
& \left.-\beta\left(\frac{r_{p q}}{R_{p q}^{\mathrm{vdW}}}\right)^{\beta} \exp \left[-\left(\frac{r_{p q}}{R_{p q}^{\mathrm{vdW}}}\right)^{\beta}\right]\right) \\
& +\left(\beta\left(\frac{r_{p q}}{R_{p q}^{\mathrm{vdW}}}\right)^{\beta}+1-\beta\right) \beta\left(\frac{r_{p q}}{R_{p q}^{\mathrm{vdW}}}\right)^{\beta} \\
& \times \frac{r_{p q}^{a} r_{p q}^{b}}{r_{p q}^{5}} \exp \left[-\left(\frac{r_{p q}}{R_{p q}^{\mathrm{vdW}}}\right)^{\beta}\right] .
\end{aligned}
$$

The interaction energy is given by the difference of the coupled and uncoupled systems of QHOs,

$$
E_{\mathrm{MBD}}=\frac{1}{2} \sum_{i=1}^{3 N} \sqrt{\lambda_{i}}-\frac{3}{2} \sum_{p=1}^{N} \omega_{p},
$$

where $\left\{\lambda_{i}\right\}$ are the eigenvalues of the matrix $C^{\mathrm{QHO}}$ (Eq. (6)). Within the approximations of the model, the MBD energy includes all contributions of the dispersion interaction, including the pairwise component.

\section{Molecular polarizability}

The MBD eigenvalue problem (Eq. (6)) has been shown equivalent to the calculation of molecular polarizability from a dipole-dipole electric field coupling equation. ${ }^{35}$ As described by Applequist ${ }^{36}$ the molecular polarizability can be obtained from a combination of the eigenvalues and eigenvectors of Eq. (6), the norm of eigenvector $i$ being subsequently scaled by $\alpha_{i}^{-1 / 2} \omega_{i}^{-1}$. Though all atomic polarizabilities and characteristic frequencies considered up to now were purely isotropic, we have found strongly enhanced molecular polarizability anisotropies when using anisotropic coefficients for the eigenvector scaling. To do so, we replace the $r^{3}$ factor in Eq. (3) by $\left(\mathbf{r} \cdot \mathbf{e}_{\alpha}\right)^{2} r$, where $\mathbf{e}_{\alpha}$ is a Cartesian unit vector along $\alpha \in\{x, y, z\}$.

\section{TBD energy}

While the reward for using MBD is evident, inclusion of TBD in the discussion also has its merits. The TBD amounts to (i) a commonly used DFT correction, (ii) the dissociative Lennard-Jones tail employed for intermolecular non-covalent energy contributions in many force-fields, (iii) an interesting comparison of two-body versus many-body effects, and (iv) a straightforward way to evaluate analytic force contributions. As such, our TBD implementation suggests a simple recipe to approximately include the dynamic, through atomic Voronoi partitioning re-evaluated "on-the-fly," dispersion coefficients in MD packages, as it only requires the estimation of effective polarizabilities to determine the $C_{6}$ coefficients. Therefore, and following the TS prescription, ${ }^{20}$ we also extend the discussion to include dispersion energies arising from exclusively pairwise dipole-dipole interactions,

$$
E_{\mathrm{TBD}}=-\frac{1}{2} \sum_{p, q} f_{\mathrm{damp}}\left(r_{p q}, R_{p}^{\mathrm{vdW}}, R_{q}^{\mathrm{vdW}}\right) C_{6 p q} r_{p q}^{-6},
$$

where

$$
C_{6 p q}=\frac{2 C_{6 p p} C_{6 q q}}{\frac{\alpha_{q}}{\alpha_{p}} C_{6 p p}+\frac{\alpha_{p}}{\alpha_{q}} C_{6 q q}},
$$

the $C_{6}$ and $\alpha$ parameters are computed from the frequencydependent polarizability $\alpha(i \omega)$, and we use a Fermi-type damping function

$$
f_{\text {damp }}\left(r_{p q}, R_{p}^{\mathrm{vdW}}, R_{q}^{\mathrm{vdW}}\right)=\frac{1}{1+\exp \left[-d\left(\frac{r_{p q}}{s_{R}\left(R_{p}^{\mathrm{vdW}}+R_{q}^{\mathrm{vdW}}\right)}-1\right)\right]},
$$

where $d$ and $s_{R}$ are unitless, free parameters obtained through optimization (see below).

\section{E. Multipole electrostatics}

Electrostatic interactions were computed using a static multipolar expansion. The electrostatic potential (ESP), $\Phi(\mathbf{r})$ $=\int \mathrm{d} \mathbf{r}^{\prime} n\left(\mathbf{r}^{\prime}\right) /\left|\mathbf{r}-\mathbf{r}^{\prime}\right|$, of a charge density $n$ at position $\mathbf{r}$ is expanded in a Taylor series of $1 / R \equiv 1 /\left|\mathbf{r}-\mathbf{r}^{\prime}\right|$, providing the following MTP expansion for $\Phi$ in Cartesian coordinates:

$$
\Phi(\mathbf{r})=\frac{q}{R}+\frac{\mu_{\alpha} R_{\alpha}}{R^{3}}+\frac{1}{3} \Theta_{\alpha \beta} \frac{3 R_{\alpha} R_{\beta}-R^{2} \delta_{\alpha \beta}}{R^{5}}+\cdots,
$$

where $q$ is a partial charge, $\mu_{\alpha}$ is the component $\alpha$ of the dipole moment $\mu, \Theta_{\alpha \beta}$ is the component $\alpha \beta$ of the secondrank quadrupole moment tensor $\Theta$, and summation over repeating indices is implied. Analogous to Coulomb interactions, the MTP interaction is determined by the work done on a MTP $Q_{l \kappa}$ (i.e., order $l$ and index $\kappa$ ) brought from infinity to a point $r$ in a region populated by the potential $\Phi, E_{\mathrm{MTP}}=\Phi Q_{l \kappa} \cdot{ }^{37}$ For this study, MTP coefficients up to quadrupoles have been placed on each atomic site. All 
multipole interactions were computed in $\mathrm{CHARMM}^{38}$ using the MTPL module. ${ }^{39,40}$

\section{RESULTS}

\section{A. Parameter optimization}

\section{Multipole coefficients}

MTP coefficients were obtained by means of a fit to the $a b$ initio ESP of each compound, using second-order Møller Plesset and an aug-cc-pVDZ basis set (except for the supramolecular host-guest complexes, see below). For each molecule, we computed the $a b$ initio ESP of the conformation provided by the reference geometry, without further optimization. MTP parameters were then fitted to best reproduce the ESP in the close vicinity of the molecule (see Eq. (13)). To avoid fitting artifacts due to insufficient sampling of buried atoms, we constrained each monopole to deviate at most by an amount $0.1 e$ from the corresponding monopole derived from the generalized distributed multipole analysis (GDMA). ${ }^{37}$ More details on the ESP-based fitting protocol, as well as scripts to reproduce the present results, can be found in Kramer et al. ${ }^{30}$

\section{Polarizabilities}

The estimation of molecular polarizabilities based on Voronoi partitioning requires the determination of three free parameters: (i) $d_{w}$, which controls the decay of the weight $w_{p}(\mathbf{r})$ in the polarizability calculation (Eq. (3)), (ii) $\beta$ from the modified Coulomb potential (Eq. (7)), and (iii) the radius prefactor $\gamma_{\mathrm{MBD}}$ (see Sec. II B). We have found, however, that the latter two affect molecular polarizabilities negligibly, within a reasonable range-they will be parametrized in Sec. III A 3. We optimize $d_{w}$ globally, i.e., it is not atom-type dependent, to best reproduce a set of 18 experimentally determined molecular polarizabilities benchmarked in Refs. 41, 19, and 22. The parameter was systematically varied to minimize the mean-absolute relative error (MARE: $\left.\sum_{i}\left|\alpha_{i}^{\text {exp }}-\alpha_{i}^{\text {model }}\right| / \alpha_{i}^{\text {exp }}\right)$ of the isotropic molecular polarizabilities. For $d_{w}=3.8 \mathrm{Bohr}^{-1}$, we find minimal MARE values of $6 \%$ for the isotropic polarizabilities. This outcome compares favorably with the $9.1 \%$ MARE found by DiStasio et $a .^{22}$ Fractional anisotropies (FA: $1 / 4\left\{\left[\left(\alpha_{x x}-\alpha_{y y}\right)^{2}\right.\right.$ $\left.\left.\left.+\left(\alpha_{x x}-\alpha_{z z}\right)^{2}+\left(\alpha_{y y}-\alpha_{z z}\right)^{2}\right] /\left(\alpha_{x x}^{2}+\alpha_{y y}^{2}+\alpha_{z z}^{2}\right)\right\}^{1 / 2}\right) \quad$ are not as well accounted for: $68 \%$, while they found $34 \%$. Fig. 1 shows the scatter plot of (a) the isotropic molecular polarizabilities and (b) fractional anisotropies predicted from the best-performing parameters versus experimental values used for fitting. We find decent correlation for the fractional anisotropies against the experimental values, though our model systematically underestimates them. This systematic effect hints at an imbalance in the dipole interaction tensor, though none of its free parameters $\left(\gamma_{\mathrm{MBD}}\right.$ and $\beta$ ) affects significantly the fractional anisotropies. The more pronounced effect of $d_{w}$ suggests the role of the quality of the atomic polarizabilities themselves. A simple Voronoi partitioning
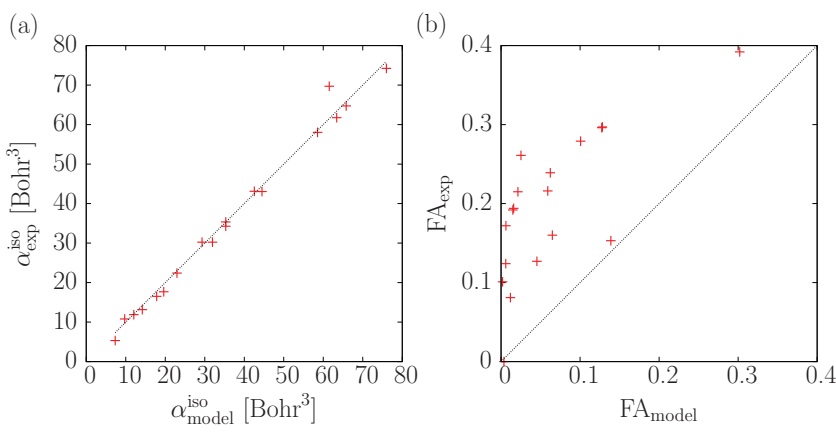

FIG. 1. Correlation plot between (a) isotropic and (b) fractional anisotropies of molecular polarizabilities predicted from the current implementation and experimental values for the set of 18 compounds proposed in Ref. 41 .

scheme is likely not sufficient to appropriately describe the anisotropy of molecular polarizabilities.

\section{MBD interaction energies}

To compute relaxed geometries within MBD, two additional parameters required optimization, the range-separation parameter, $\beta$, of the modified Coulomb potential (Eq. (7)), and the radius prefactor $\gamma_{\mathrm{MBD}}$ that scales $R_{p q}^{\mathrm{vdW}}$ (see Sec. II B). These parameters were systematically varied by adding up MBD and MTP energies to best reproduce the set of intermolecular dimer energies from the S22 dataset ${ }^{42}-$ a benchmark database with 22 high-level quantum chemistry (coupled cluster with single and double and perturbative triple excitations (CCSD(T)) ) interaction energies for dimers of small molecules, DNA base pairs, and amino acid pairs in their minimal potential energy geometry. In this regard, the MTP intermolecular energies are assumed to be correct and subtracted from the reference energies, such that we optimize the free parameters to minimize the quantity $\sum_{i} \mid E_{i}^{\mathrm{MBD}}-$ $\left(E_{i}^{\text {ref }}-E_{i}^{\mathrm{MTP}}\right) \mid$. The values $\beta=1.10$ and $\gamma_{\mathrm{MBD}}=1.85$ yield a minimal mean-absolute error (MAE: $\sum_{i}\left|E_{i}^{\text {ref }}-E_{i}^{\text {model }}\right|$ ) of $1.7 \mathrm{kcal} / \mathrm{mol}$. It is noteworthy that a number of common DFT functionals were found to perform at similar level or worse. ${ }^{43}$ Combination of MBD with PBE0 has been shown to yield an error of only $0.3 \mathrm{kcal} / \mathrm{mol}^{19}$ Our results for $\mathrm{S} 22$ also compare well to energies predicted by a number of standard force fields, Amber $(2.1 \mathrm{kcal} / \mathrm{mol})$, OPLS-AA $(2.0 \mathrm{kcal} / \mathrm{mol})$, and MMFF94s (1.6 kcal/mol). ${ }^{44}$

To analyze the performance of our MBD+MTP model, we display in Fig. 2 the energy decomposition between MTP and MBD together with the reference energies for each compound in the S22 dataset. As one would expect, for the hydrogen-bonded dimers, the MTP contributions dominate, with the MBD contribution typically improving the overall prediction. The most dramatic failure for this class of dimers is found in the case of the 2-pyridoxine 2-aminopyridine complex, where MBD+MTP underestimates the actual interaction energy by $\sim 10 \mathrm{kcal} / \mathrm{mol}$. At this point, we also stress the lack of higher multipoles (i.e., beyond dipole) in the MBD method (e.g., $C_{8}, C_{10}$ coefficients). Jones et $a .^{45}$ have recently summarized the types of dispersion interactions that arise from the different induced multipoles. This error might also originate from the lack of induced multipole moment contributions, 


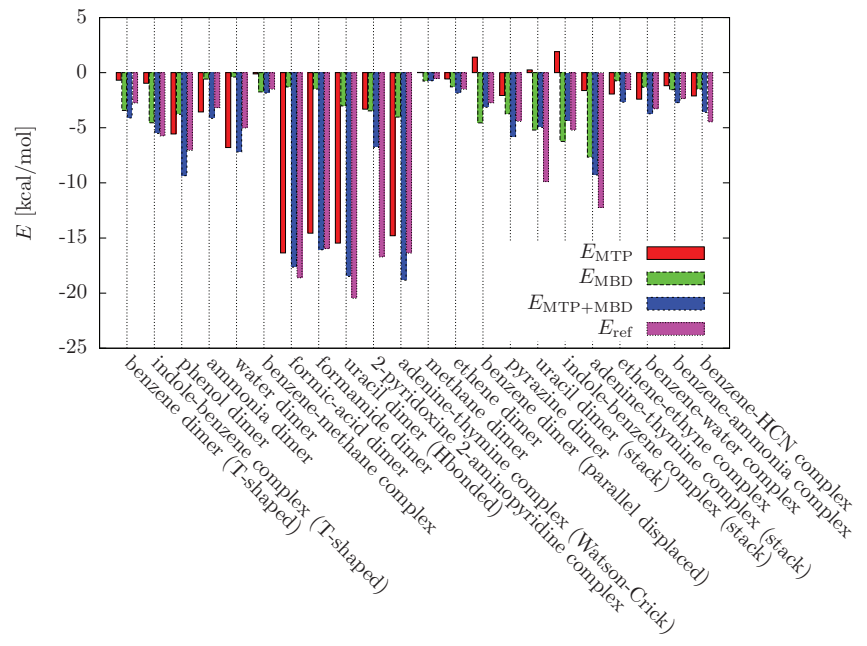

FIG. 2. Intermolecular energy decomposition of the S22 dataset. MTP, $\mathrm{MBD}, \mathrm{MTP}+\mathrm{MBD}$, and $E_{\text {ref }}$ stand for multipole electrostatic, many-body dispersion, and reference energy $(\mathrm{CCSD}(\mathrm{T}))$ contributions. ${ }^{42}$ This dataset has been used to optimize the MBD parameters in our model.

which are not accounted for within our model. Another possible source of errors could be due to the fact that in this work, we rely on the MBD energy alone to fully reproduce all dispersion at short as well as long-range interatomic distances. A $\beta$-value smaller than 2 will yield a finite amount of dispersion energy in the zero-distance limit. We note that in contrast the augmentation of DFT functionals with dispersion corrections typically implies $\beta \geq 2$, i.e., $W\left(r_{p q} \rightarrow 0\right) \rightarrow 0$, due to some amount of dispersion energy already accounted for through the short range correlation in the functional. ${ }^{19}$ Clearly, also capturing short-range correlation effects still leaves room for further improvement. In the case of the dimers where the interaction energy is dominated by dispersion, the combination of MTP and MBD predicts interaction energies that compare very favorably with the reference numbers. It is particularly encouraging that in several cases an overestimation due to MBD is offset by repulsive MTP contributions resulting in an accurate overall interaction energy, e.g., indole-benzene complex (stack). We also note that the entire MBD contribution is attractive in nature for all the instances in the $\mathrm{S} 22$.

\section{TBD interaction energies}

In the same spirit as for MBD, TBD energies are compared to the reference energies, subtracted by the MTP contribution. Errors in TBD energies in the S22 relaxed geometries were minimized through tuning of the dimensionless free parameters $d$ and $s_{R}$ (Eq. (12)). While $d=20.0$ is a commonly used value, ${ }^{20,46,47}$ we have found $d=11.0$ to perform best. As for $s_{R}$, a value of 2.20 proved to be best. Overall, the error converges to a MAE of $2.0 \mathrm{kcal} / \mathrm{mol}$, which also compares extremely favorably against other DFT functionals without dispersion correction and standard force fields.

\section{5. $C_{6}$ coefficients from Voronoi partitioning}

To assess the performance of the Voronoi partitioning scheme for estimating $C_{6}$ coefficients of atoms in molecules,

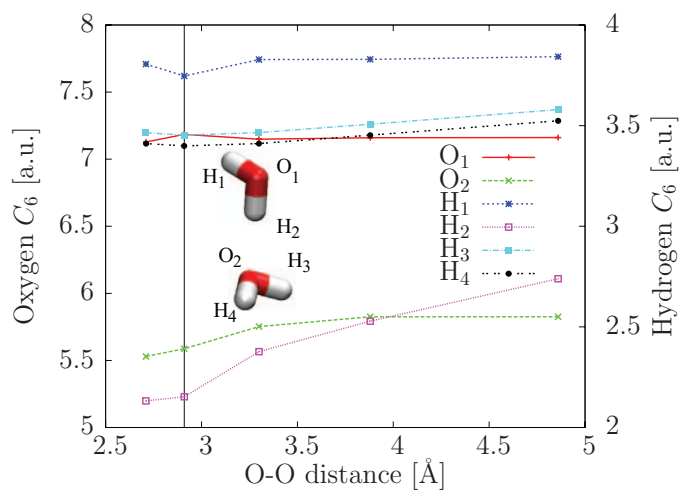

FIG. 3. $C_{6}$ coefficients obtained from Voronoi partitioning (Eq. (2)) for all hydrogen and oxygen atoms in the water dimer as a function of the oxygenoxygen distance. $C_{6}$ coefficients in units Hartree Bohr ${ }^{6}$.

we compute the $C_{6}$ coefficients of the water dimer in the S22 dataset as a function of oxygen-oxygen distance. Specifically, the water dimer exhibits $C_{6}$ values of $\approx 3$ and 7 Hartree Bohr ${ }^{6}$ for the hydrogen and oxygen atoms, respectively, as shown in Fig. 3. We find slightly large and low $C_{6}$ values for hydrogen and oxygen atoms, respectively, compared to higher-level calculations. ${ }^{16,20,48}$ We suggest that our simple Voronoi partitioning scheme does not sufficiently account for the difference in electronegativity of the two atoms, in spite of the partitioning's dependence to the free-atom's van der Waals radii. To further probe the $C_{6}$ coefficients obtained from Voronoi partitioning, we computed their average values for each chemical element across the $\mathrm{S} 22$ dimers. We find the following: $\mathrm{C}$ (15.3 $\pm 0.4), \mathrm{O}(7.1 \pm 0.9), \mathrm{N}(8.4 \pm 1.3)$, and $\mathrm{H}(3.1 \pm 0.4)$, all expressed in Hartree Bohr ${ }^{6}$. Reference values are in the ranges: $\mathrm{C}(24-33), \mathrm{O}(12-15), \mathrm{N}(17-20)$, and $\mathrm{H}(2-3)$, also in atomic units. ${ }^{20}$ Still, the geometry-dependent variation of the $C_{6}$ coefficients is properly accounted for and illustrates the coupling between the two molecules.

\section{Molecular dimers}

In Table I, we report the MAE of potential energies of interaction for a total of over 1300 geometries of small molecular dimers drawn from a variety of databases designed to probe non-covalent interactions (extracted from Ref. 49).

TABLE I. MAE of TBD, MBD, and MTP contributions to intermolecular energies of binding for various geometries containing a grand total of over 1300 dimers. All values are in $\mathrm{kcal} / \mathrm{mol}$.

\begin{tabular}{lcrrcc}
\hline \hline & MTP & TBD & MBD & TBD+MTP & MBD+MTP \\
\hline S22 & 3.46 & 4.71 & 4.87 & 2.04 & 1.67 \\
S22 $\times 5$ & 2.63 & 3.88 & 3.25 & 2.94 & 1.90 \\
S66 & 2.43 & 3.91 & 3.42 & 3.19 & 2.72 \\
S66 $\times 8$ & 2.05 & 3.72 & 2.81 & 3.12 & 2.08 \\
IonicHb & 2.53 & 15.07 & 15.13 & 2.52 & 2.62 \\
SCAI & 3.32 & 14.76 & 13.06 & 5.83 & 2.88 \\
X40 & 2.71 & 2.67 & 2.56 & 2.11 & 2.00 \\
X40 $\times 10$ & 2.85 & 2.71 & 2.26 & 3.22 & 2.83 \\
Average & 2.75 & 6.43 & 5.92 & 3.12 & 2.34 \\
\hline
\end{tabular}


These complexes include the $\mathrm{S} 22{ }^{42}$ its extension to nonequilibrium geometries, $\mathrm{S} 22 \times 5,{ }^{50} \mathrm{~S} 66$ - a larger coverage of interactions found in organic molecules and biomolecules-and its extension to nonequilibrium geometries, $\mathrm{S} 66 \times 8,{ }^{51}$ hydrogen bonds found in ionic groups interacting with neutral compounds (IonicHb) - constructed in analogy to $\mathrm{S} 66 \times 8{ }^{.52}$ a set of 24 pairs of representative amino acid side chain interactions (SCAI) ${ }^{53}$ a set of 40 complexes of organic halides, halohydrides, and halogen molecules at equilibrium (X40) and nonequilibrium $(\mathrm{X} 40 \times 10)$ geometries. ${ }^{54}$ Results are shown in Table I for the respective MTP, TBD, and MBD contributions. We remind the reader that only S22 was used to fit the free parameters of the method. As one would expect, the sum of dispersion and electrostatics (referred to as "MBD+MTP") performs on average better than any individual components, except in a few isolated cases (i.e., see components of S66, $\mathrm{S} 66 \times 8$, and IonicHb). In the case of S22, MBD+MTP performs on par with standard force fields for the SCAI database: OPLS $(2.1 \mathrm{kcal} / \mathrm{mol})$ and Amber FF03 $(2.2 \mathrm{kcal} / \mathrm{mol}){ }^{53}$ The improvement for going from TBD to MBD is largest for the SCAI-database, possibly due to the aromaticity of a number of amino-acid side chains.

A scatter plot between all 1300 intermolecular energies predicted from the current implementation and reference energies (most of which are $\operatorname{CCSD}(\mathrm{T})$ ) is shown in Fig. 4. Clearly, the $\mathrm{X} 40$ and $\mathrm{X} 40 \times 10$ data sets represent the most difficult challenges for MBD+MTP. The MBD+MTP severely overestimates the binding of $\mathrm{HCl}$-methylamine, $\mathrm{HF}-$ methylamine, HF-methanol, trichloromethanol-water, and trifluoromethanol-water-specifically for distances shorter than the equilibrium geometry, where repulsive interactions are critically lacking from our model (see also Fig. 5). For those, the MTP energy alone is significantly lower than the reference energy. Similar overestimations are also encountered in the case of the larger supramolecular guest-host systems discussed below, though the role of MTP there is not as clear.

To further analyze the quality of our approach, we decomposed the MAEs of the $\mathrm{S} 22 \times 5$, S66 $\times 8$, IonicHb, and $\mathrm{X} 40 \times 10$ datasets at each distance factor that is used to scale

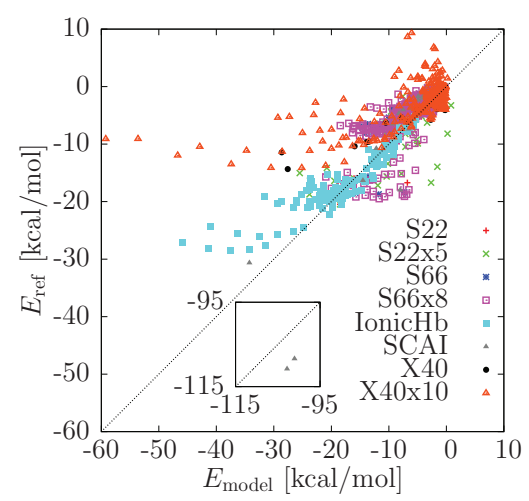

FIG. 4. Correlation plot between energies predicted from the current implementation, $E_{\text {model }}=\mathrm{MBD}+\mathrm{MTP}$, and reference energies, $E_{\text {ref }}$ for each database (Table I). The inset corresponds to two charged-charged side-chain interactions of the SCAI database. Strong outliers from X40 $\times 10$ exert overstabilizing MTP interactions (see main text).
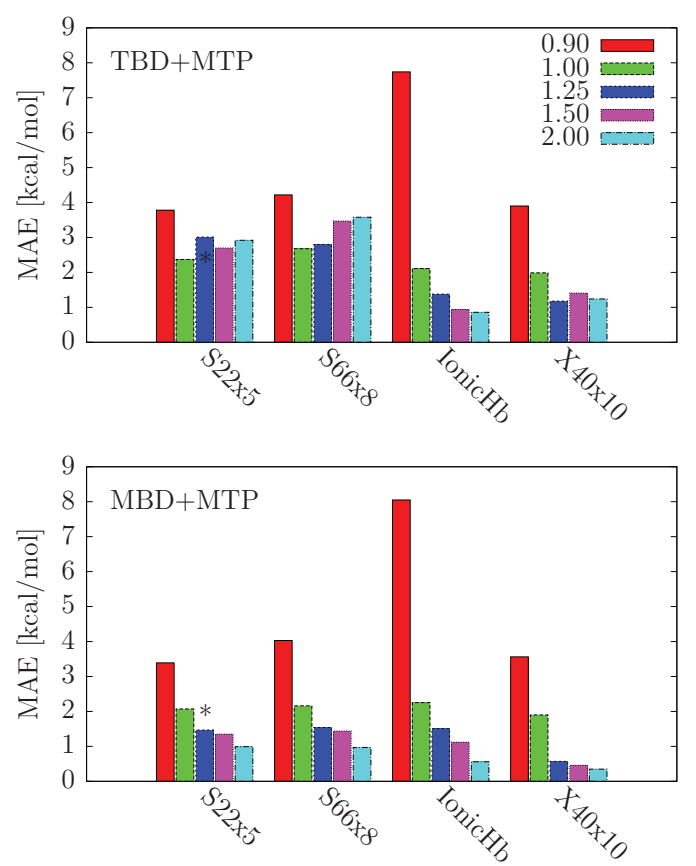

FIG. 5. MAE as a function of distance relative to the equilibrium geometry for the databases $\mathrm{S} 22 \times 5, \mathrm{~S} 66 \times 8$, IonicHb, and $\mathrm{X} 40 \times 10$ for both TBD+MR (top) and MBD+MR (bottom). The intermolecular distance at equilibrium geometry is scaled by a factor (i.e., 1.00 corresponds to the equilibrium geometry). Note that the $\mathrm{S} 22 \times 5$ at 1.25 is referring to an energy that was in fact calculated using a factor of 1.20 instead (denoted by asterisks above the corresponding histograms).

their nonequilibrium geometries (i.e., at $0.90,1.00,1.25,1.50$, 2.00). This decomposition is shown for both TBD+MTP and MBD+MTP in Fig. 5. For MBD+MTP, we find the deviation from reference systematically decaying with the intermolecular distance for all the four datasets. This underscores the fact that the correct dissociative asymptotics has been taken into account via the MBD. For smaller distances, however, the lack of Pauli- and Coulomb-repulsion in our model appears to be at the origin of an increasing error. For the TBD+MTP data, however, no such physically correct behavior is found for larger distances. In the case of S22 and S66, the error at twice the equilibrium distance $(\sim 7 \AA)$ is in fact as large as the error at 0.9 of the equilibrium distance. This finding conflicts with the common assumption that the simple $C_{6} / R^{6}$ correction will yield proper dissociative tails. While hardly relevant for dimers in gas-phase, one can expect this effect to become very significant in crowded or condensed-phase scenarios where second neighbor solvation shells contribute significantly to the cohesive energy. We note that the low errors for TBD+MTP in the IonicHb and $\mathrm{X} 40 \times 10$ datasets are the result of a relatively small weight of the TBD interactions compared to electrostatics. As such, the small error at large distances is mainly associated to the electrostatics that has well been accounted for through MTP.

\section{Water clusters}

Moving toward more complex systems, we further benchmarked the present method against a series of 38 water clusters containing 2-10 water molecules. ${ }^{55}$ The 


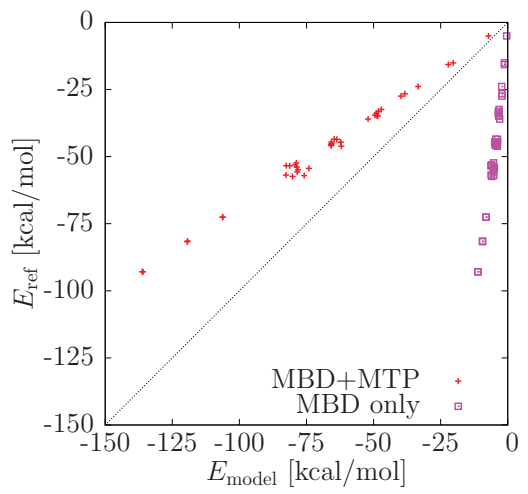

FIG. 6. Predicted interaction energies from the current implementation, $E_{\text {model }}=\mathrm{MBD}+$ MTP versus reference energies, $E_{\text {ref }}$, for 38 water clusters containing 2 up to 10 monomers, respectively. The "MBD only" dataset displays the MBD component alone: Most of the stabilization energy arises from electrostatics.

importance of many-body dispersion in small water clusters has been highlighted by Gillan et al. ${ }^{56}$ Santra et al. ${ }^{57,58}$ studied similar water clusters and reported errors that were within $1 \mathrm{kcal} / \mathrm{mol}$ of reference $a b$ initio data using PBE0. The results of our approach, shown in Fig. 6, indicate a strong correlation between MBD+MTP energies versus reference CCSD(T) $a b$ initio data over a large energy range. As the cluster increases in size, the estimates become overstabilizing. For all clusters but the smallest ones, we consistently find an error of $\approx 4 \mathrm{kcal} / \mathrm{mol}$ per water molecule. The MBD component is comparatively weak, as shown in Fig. 6, and illustrates that the MTP interaction alone overstabilizes the complex. Defusco et al. ${ }^{59}$ highlighted the importance of repulsion to accurately describe water-cluster geometries. Polarizable electrostatics is also known to play an important role, ${ }^{60,61}$ though it is likely to lower the binding energy even further. ${ }^{62}$ Interestingly, our MTP coefficients are in good agreement with the original AMOEBA water model. ${ }^{62}$ Their excellent results in reproducing small water clusters further suggests the role of repulsion in this discrepancy.

\section{Benzene crystal}

To further assess the performance of our approach, we computed the cohesive binding energy of the benzene crystal. Crystal structure prediction of organic compounds has benefitted from steady progress, owing in part to the development of both fast and accurate modeling methods (e.g., dispersion-corrected DFT), ${ }^{63}$ to the point of ranking polymorphs of molecular crystals. ${ }^{24}$ Akin to Refs. 11 and 65, we computed the binding energy for different ratios of $\rho / \rho_{\text {exp }}$, where $\rho$ denotes the unit-cell density and the experimental value is taken from Ref. 66. Density scalings were performed isotropically, while the monomers of the unit cell were translated relative to the corresponding scaling of their center of mass. ${ }^{64}$ As a result, we restrained our study to small deviations, i.e., $\left|\rho / \rho_{\exp }\right| \leq 5 \%$. The binding energy was computed using $E_{\text {binding }}=\left(E_{\text {complex }}-\sum_{i=1}^{N} E_{\text {monomer }, i}\right) / N$, where $N$ is the number of monomers in the unit cell (i.e., $N=4$ in this case). Fig. 7 shows the binding energy as a function of $\rho / \rho_{\exp }$.

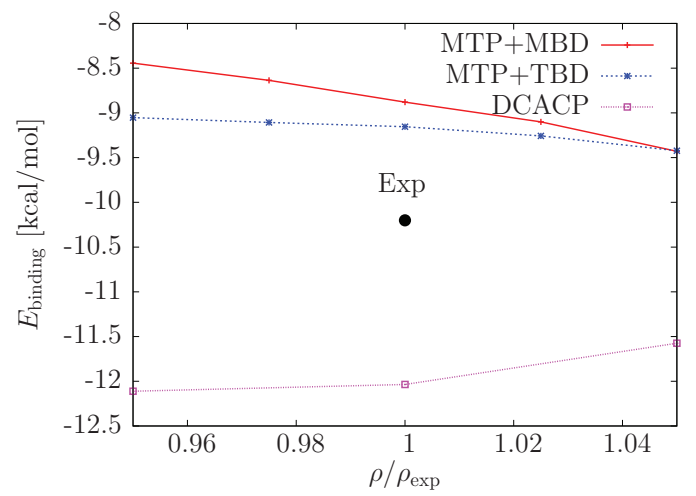

FIG. 7. Cohesive binding energy of the benzene crystal as a function of the ratio of densities, $\rho / \rho_{\exp }$. The experimental value is shown explicitly (black dot). ${ }^{66}$ The DCACP data correspond to the BLYP + DCACP-CCSD(T) calculations of Tapavicza et al. ${ }^{65}$

We find very good agreement with the experimental energy (i.e., $E_{\text {binding }}^{\exp }=-10.2 \mathrm{kcal} / \mathrm{mol}$ ). In most cases, the MTP and MBD energy contributions are $25 \%$ and $75 \%$, respectively. While Fig. 7 indicates a monotonously decreasing curve, an energy minimum is expected due to the rising contribution of repulsion as $\rho$ gets larger. The corresponding TBD results are on par (see Fig. 7). For the sake of comparison, we plot the results of Tapavicza et al., ${ }^{65}$ who used dispersion corrected atom centered potentials (DCACP) in combination with the BLYP density functional.

\section{Supramolecular complexes}

As the final and most challenging test-case, we considered several supra-molecular host-guest complexes where one moiety encloses to a very large degree the structure of the other moiety. First, we calculated the interaction energy for two Watson-Crick-bound DNA base pairs, connected through charged sugar-phosphate backbone, into which ellipticine is intercalated (Fig. 8(a)). The binding energy was estimated to amount to $\approx 37 \mathrm{kcal} / \mathrm{mol}$ from a dispersion-corrected DFT method, ${ }^{67} 48 \mathrm{kcal} / \mathrm{mol}$ from the Tkatchenko-Scheffler method (i.e., without subsequent $\mathrm{SCS}),{ }^{16} 50.7 \mathrm{kcal} / \mathrm{mol}$ from the non-range-separated MBD method with DFT, ${ }^{23}$ and $33.6 \pm 0.9 \mathrm{kcal} / \mathrm{mol}$ from diffusion quantum Monte Carlo. ${ }^{68}$ The complex has significant $\pi-\pi$ stacking and many-body effects. ${ }^{16}$ We find a binding energy $E_{\text {binding }}^{\mathrm{MBD}}=60 \mathrm{kcal} / \mathrm{mol}$, where the MTP contribution is destabilizing (i.e., $+7.4 \mathrm{kcal} / \mathrm{mol}$; see Table II). We further note that the binding energy using TBD is significantly stronger: $E_{\text {binding }}^{\mathrm{TBD}}=182 \mathrm{kcal} / \mathrm{mol}$. These trends are in qualitative agreement with previous results, ${ }^{16}$ though they suggest that, as already seen for larger systems above, the current MBD implementation overestimates the binding energy as the contact area between moieties grows.

Second, we also studied a select set of six hostguest supramolecular complexes (Figs. 8(b)-8(g)) extracted from the S12L database of Grimme. ${ }^{70,71}$ These complexes are formed by a host molecule (e.g., "tweezer," "pincers") and a guest (i.e., small organic molecule) stabilized by non-covalent interactions, including vdW interactions, 
(a)
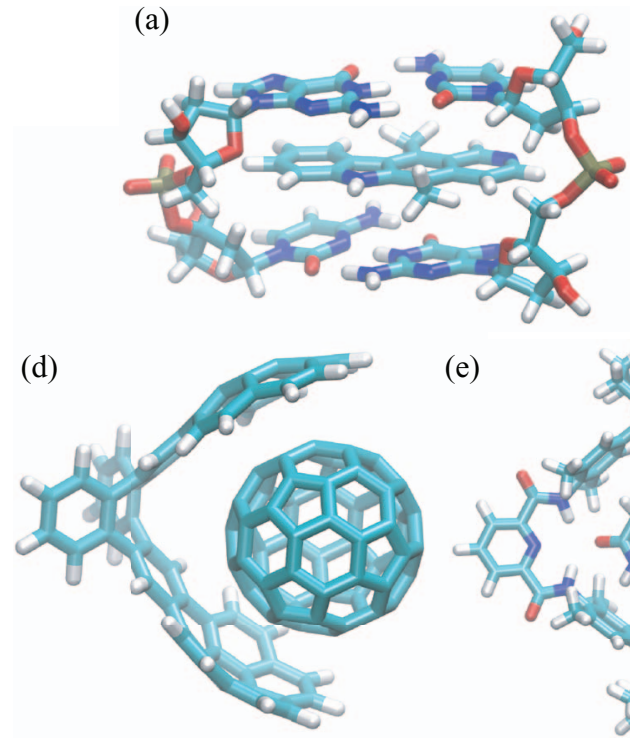

(e)

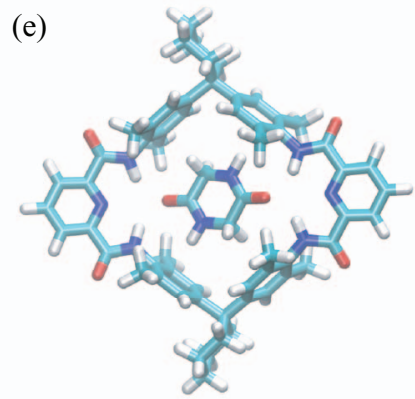

(b)

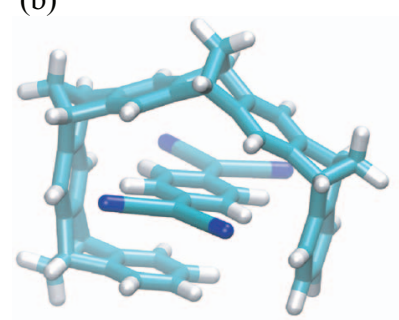

(f)

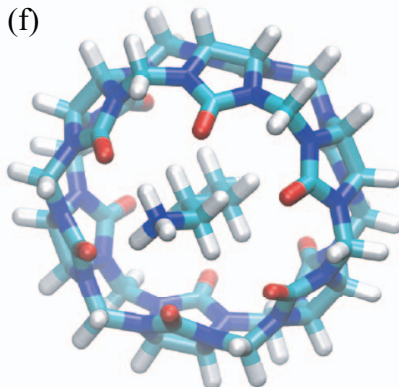

(c)

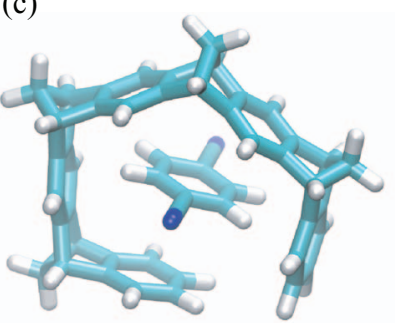

(g)

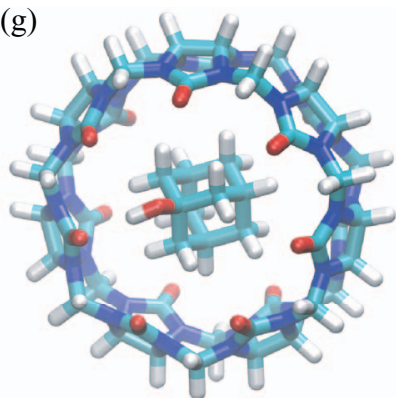

FIG. 8. Cartoon representations of (a) the intercalating ellipticine between two Watson-Crick-bound DNA base pairs, (b) tetracyanoquinone-tweezer, (c) 1,4dicyanobenzene-tweezer, (d) buckyball-catcher, (e) glycine anhydride-macrocycle, (f) butylammonium-cucurbit[6]uril cation, and (g) 1-hydroxyadamantanecucurbit[7]uril. Rendered in VMD. ${ }^{69}$

hydrogen-bonding, $\pi-\pi$ stacking, and electrostatic attraction. The subset of complexes selected here follows a recent study where diffusion quantum Monte Carlo reference numbers have been obtained: ${ }^{72}$ tetracyanoquinone-tweezer (b), 1,4dicyanobenzene-tweezer (c), buckyball-catcher (d), glycine anhydride-macrocycle (e), butylammonium-cucurbit[6]uril cation (f), and 1-hydroxyadamantane-cucurbit[7]uril (g). Because of the sheer size of these systems (each host contained between 72 and 130 atoms), the $a b$ initio calculations devoted to the ESP-based fitting were run with an M06-2X functional $^{73}$ with $6-31+\mathrm{G}(\mathrm{d}, \mathrm{p})$ basis set. Unlike the aforementioned protocol (i.e., MTP coefficients from MP2/augcc-pVDZ and monopole constraints from GDMA), we fitted the MTP coefficients of the supramolecular complexes to the $a b$ initio ESP without any monopole constraints. The resulting MTP and MBD/TBD energies are presented in Table II. Also in this case, we observe significant overstabilization of the complexes. Interestingly, the MBD interactions are always less attractive than their TBD counterparts. Different complexes show various electrostatic contributions (e.g.,

TABLE II. Energy contributions and reference energies (from diffusion quantum Monte Carlo ${ }^{68,72}$ ) for the ellipticine drug (a) and the six supramolecular complexes (b)-(g). The last line indicates the MAE of each field against the reference values. All energies are in $\mathrm{kcal} / \mathrm{mol}$.

\begin{tabular}{lcccccc}
\hline \hline & MTP & TBD & MBD & TBD+MTP & MBD+MTP & Ref. \\
\hline (a) & +7.36 & -189 & -67.0 & -182 & -59.6 & -33.6 \\
(b) & -13.1 & -69.8 & -36.5 & -82.9 & -49.6 & -27.5 \\
(c) & -6.51 & -47.1 & -26.4 & -53.6 & -32.9 & -17.2 \\
(d) & +0.14 & -224 & -81.5 & -224 & -81.3 & -25.8 \\
(e) & -34.6 & -68.7 & -29.6 & -103 & -64.2 & -33.4 \\
(f) & -63.6 & -72.5 & -32.6 & -136 & -96.2 & -81.0 \\
(g) & -0.01 & -142 & -54.3 & -142 & -54.4 & -24.1 \\
MAE & 19.2 & 83.9 & 27.1 & 97.3 & 27.9 & \\
\hline \hline
\end{tabular}

the buckyball catcher includes almost none). This finding represents considerable numerical evidence for suggesting that a proper inclusion of repulsive interactions and induced electrostatics becomes all the more important as we probe more condensed or crowded environments and scenarios. Overall, we observe similar overstabilization as for the water clusters (above). Given that an accurate account of MBD and MTP contributions significantly overstabilizes intermolecular energies, and that induced electrostatics systematically strengthens the binding energy, an additive force field will require enhanced repulsive interaction terms. Interatomic repulsion originates in the interplay of Coulomb-repulsion of electronselectrons and nuclei-nuclei, as well as Pauli exchange. As such, it appears unlikely that it can be modeled through a purely pair-wise effective potential.

\section{CONCLUSION}

We introduced a Voronoi partitioning scheme for the construction of polarizabilities of atoms in molecules, effectively coarse-graining away the electron density. Accounting for the heterogeneity of atoms through their free-atom van der Waals radii provides the means to partition atoms in molecules via a simple yet effective scheme. Its ability to reproduce molecular polarizabilities within the many-body-dispersion scheme, ${ }^{19}$ as well as interaction energies when combined with multipole electrostatics, makes it an appealing technique to explore a wide variety of molecular systems exhibiting noncovalent interactions. Specifically, the intermolecular energies of more than 1300 dimers were benchmarked against high-level $a b$ initio data (i.e., $\operatorname{CCSD}(\mathrm{T})$ in most cases) - the S22 dataset alone was used for parameter optimization of the two-body and many-body dispersion energies, where the electrostatic contribution was subtracted from the reference energy. The method presents an overall mean-absolute error of $\approx 2.3 \mathrm{kcal} / \mathrm{mol}$-roughly on par with standard force fields 
(e.g., OPLS, Amber FF03). We highlight the excellent performance of the TBD energies, reproducing reference data with an accuracy of $3.1 \mathrm{kcal} / \mathrm{mol}$. The ease of the Voronoi-type tessellation scheme provides a simple and efficient means to compute atomic polarizabilities from first-principles, and extract $C_{6}$ coefficients that inherently depend on the geometry of the system. However, TBD's lack of error reduction when probing increasingly larger distances makes a strong case for the use of MBD interactions.

Naturally, the combined effect of dispersion energies and MTP electrostatics still lacks repulsive interactions: several dimers, the water clusters, ellipticine with DNA base-pair dimers, and the supramolecular complexes showed significant overstabilization, while the benzene crystal showed no minimum energy around the experimental unit-cell density. A naïve attempt at introducing pairwise repulsive-only LennardJones-type potentials (i.e., Weeks-Chandler-Anderson ${ }^{74}$ ) did little in improving the agreement with reference data (not shown)-likely due to its overly simplifying functional form. A comparison of our results with the original AMOEBA water model $^{62}$ also points to the role of repulsion (modeled there via Halgren's 14-7 buffered potentials ${ }^{75}$ ), since the other major interaction term missing is polarizable electrostatics, likely to further stabilize binding. The series of exponentials used by Whitfield and Martyna for quantum Drude oscillators also stands as a promising candidate. ${ }^{76}$ We finally note that the distributed MTP methodology does not describe penetration effects, though they are unlikely to play a large role for the systems considered here.

Performance-wise, this approach yields results in between standard force fields and DFT methods. TBD interactions require the calculation of effective atomic polarizabilities using the Voronoi partitioning, which scales linearly with the number of atoms, $N$, and the eigenvalue MBD equation will scale like $N^{3}$. In comparison, while force fields scale like $N$, DFT scales between $N_{e}^{2} \ln N_{e}$ and $N_{e}^{3}$, where $N_{e}$ is the number of electrons in the system. The computation of any complex took a fraction of a second (slightly more for several supramolecular hosts) on a single modern CPU core. It stands to reason that a number of obvious optimizations (e.g., parallelization) could be undertaken to further reduce the computational investment. We point out that the present work achieves classical intermolecular energies with similar accuracies as standard force fields, without experimental inputas achieved earlier by other methods ${ }^{25,27}$ —and negligible parametrization effort: the TBD/MBD energy has no free parameter (excluding the transferable parameters that were set in this work) irrespective of the chemistry at hand (i.e., no need for specific atom types) - though heavier elements may present additional complications-while systematic protocols can fit MTP coefficients from the $a b$ initio ESP in an automated way. We further note that this prior ab initio calculation is not a necessary curse: an optimized workflow could involve a fragment-based library of precomputed MTP coefficients. The limited computational overhead associated with the TBD method would make its use in force fields conceivable: the inherent dependence of the $C_{6}$ coefficients on the geometry and chemistry (e.g., hybridization state) of the system may help reach more transferable force fields-a critically lacking feature of current atomistic models, which require extensive effort for any new (i.e., yet unparametrized) molecule studied. As for MBD, the eigenvalue equation involved hampers the prospects of an efficient MD implementation. However, it provides interesting perspectives in the context of wellestablished potentials (e.g., SIBFA, ${ }^{26} \mathrm{EFP}^{27}$ ) that aim at computing accurate intermolecular energies for large systems.

\section{ACKNOWLEDGMENTS}

We thank Alexandre Tkatchenko and Denis Andrienko for insightful discussions that shaped the present work. We also thank Sanghamitra Neogi, Davide Donadio, and Markus Meuwly for comments and critical reading of the paper. T.B. acknowledges access to Markus Meuwly's computational resources.

T.B. was partly supported by the Swiss National Science Foundation $(\mathrm{NSF}(\mathrm{CH}))$ through the NCCR-MUST and Grant No. 200021-117810. O.A.v.L. acknowledges support from the Swiss National Science Foundation Grant No. PPOOP2_138932. This research used resources of the Argonne Leadership Computing Facility at Argonne National Laboratory, which is supported by the Office of Science of the (U.S.) Department of Energy (DOE) under Contract No. DE-AC02-06CH11357.

${ }^{1}$ A. Szabo and N. S. Ostlund, Modern Quantum Chemistry: Introduction to Advanced Electronic Structure Theory, New edition (Dover Publications, 1989).

${ }^{2}$ R. G. Parr and W. Yang, Density-functional Theory of Atoms and Molecules (Oxford University Press, 1989), Vol. 16.

${ }^{3}$ R. Car and M. Parrinello, "A combined approach to DFT and molecular dynamics," Phys. Rev. Lett. 55, 2471 (1985).

${ }^{4}$ R. Iftimie, P. Minary, and M. E. Tuckerman, "Ab initio molecular dynamics: Concepts, recent developments, and future trends," Proc. Natl. Acad. Sci. U.S.A. 102, 6654 (2005).

${ }^{5}$ S. Kristyán and P. Pulay, Chem. Phys. Lett. 229, 175 (1994).

${ }^{6}$ W. Koch and M. C. Holthausen, A Chemist's Guide to Density Functional Theory, 2nd ed. (Wiley-VCH, 2002).

${ }^{7} \mathrm{O}$. Anatole von Lilienfeld, "Force correcting atom centred potentials for generalised gradient approximated density functional theory: Approaching hybrid functional accuracy for geometries and harmonic frequencies in small chlorofluorocarbons," Mol. Phys. 111(14), 2147-2153 (2013).

${ }^{8}$ K. E. Riley, M. Pitoňák, P. Jurečka, and P. Hobza, "Stabilization and structure calculations for noncovalent interactions in extended molecular systems based on wave function and density functional theories," Chem. Rev. 110(9), 5023-5063 (2010).

${ }^{9}$ R. H. French, V. A. Parsegian, R. Podgornik, R. F. Rajter, A. Jagota, J. Luo, D. Asthagiri, M. K. Chaudhury, and Y. Chiang, S. Granick et al., "Long range interactions in nanoscale science," Rev. Mod. Phys. 82(2), 1887 (2010).

${ }^{10}$ F. O. Kannemann and A. D. Becke, "van der Waals interactions in densityfunctional theory: Intermolecular complexes," J. Chem. Theory Comput. 6(4), 1081-1088 (2010).

${ }^{11}$ E. J. Meijer and M. Sprik, "A density-functional study of the intermolecular interactions of benzene," J. Chem. Phys. 105, 8684 (1996).

${ }^{12} \mathrm{~S}$. Grimme, "Accurate description of van der Waals complexes by density functional theory including empirical corrections," J. Comput. Chem. 25, 1463 (2004).

${ }^{13}$ S. Grimme, "Semiempirical GGA-type density functional constructed with a long-range dispersion correction," J. Comput. Chem. 27, 1787 (2006).

${ }^{14}$ S. Grimme, C. Diedrich, and M. Korth, "The importance of inter- and intramolecular van der Waals interactions in organic reactions: The dimerization of anthracene revisited," Angew. Chem., Int. Ed. 45, 625 (2006).

${ }^{15}$ A. Tkatchenko and O. A. von Lilienfeld, "Popular Kohn-Sham density functionals strongly overestimate many-body interactions in van der Waals systems," Phys. Rev. B 78, 045116 (2008). 
${ }^{16} \mathrm{O}$. A. von Lilienfeld and A. Tkatchenko, "Two-and three-body interatomic dispersion energy contributions to binding in molecules and solids," J. Chem. Phys. 132, 234109 (2010).

${ }^{17}$ B. Linder and D. Hoernschemeyer, "Many-body aspects of intermolecular forces," J. Chem. Phys. 40(3), 622 (1964).

${ }^{18}$ M. J. Renne and B. R. A. Nijboer, "Microscopic derivation of macroscopic Van der Waals forces," Chem. Phys. Lett. 1(8), 317 (1967).

${ }^{19}$ A. Tkatchenko, R. A. DiStasio, Jr., R. Car, and M. Scheffler, "Accurate and efficient method for many-body van der Waals interactions," Phys. Rev. Lett. 108(23), 236402 (2012).

${ }^{20} \mathrm{~A}$. Tkatchenko and M. Scheffler, "Accurate molecular van der Waals interactions from ground-state electron density and free-atom reference data," Phys. Rev. Lett. 102(7), 073005 (2009).

${ }^{21}$ A. G. Donchev, "Many-body effects of dispersion interaction," J. Chem. Phys. 125(7), 074713 (2006).

${ }^{22}$ R. A. DiStasio, Jr., V. V. Gobre, and A. Tkatchenko, "Many-body van der Waals interactions in biology, chemistry, and physics," J. Phys.: Condens. Matter 26, 213202 (2014).

${ }^{23}$ R. A. DiStasio, O. A. von Lilienfeld, and A. Tkatchenko, "Collective manybody van der Waals interactions in molecular systems," Proc. Natl. Acad. Sci. U.S.A. 109(37), 14791-14795 (2012)

${ }^{24}$ N. Marom, R. A. DiStasio, V. Atalla, S. Levchenko, A. M. Reilly, J. R. Chelikowsky, L. Leiserowitz, and A. Tkatchenko, "Many-body dispersion interactions in molecular crystal polymorphism," Angew. Chem., Int. Ed. 52, 6629-6632 (2013)

${ }^{25}$ J.-P. Piquemal, H. Chevreau, and N. Gresh, "Toward a separate reproduction of the contributions to the Hartree-Fock and DFT intermolecular interaction energies by polarizable molecular mechanics with the SIBFA potential," J. Chem. Theory Comput. 3(3), 824-837 (2007).

${ }^{26}$ N. Gresh, A. Cisneros, T. A. Darden, and J.-P. Piquemal, "Anisotropic, polarizable molecular mechanics studies of inter- and intramolecular interactions and ligand-macromolecule complexes: A bottom-up strategy," J. Chem. Theory Comput. 3(6), 1960-1986 (2007).

${ }^{27}$ M. S. Gordon, Q. A. Smith, P. Xu, and L. V. Slipchenko, "Accurate first principles model potentials for intermolecular interactions," Annu. Rev. Phys. Chem. 64, 553-578 (2013).

${ }^{28}$ J. W. Ponder, C. Wu, P. Ren, V. S. Pande, J. D. Chodera, M. J. Schnieders, I. Haque, D. L. Mobley, D. S. Lambrecht, and R. A. DiStasio, Jr. et al., "Current status of the AMOEBA polarizable force field," J. Phys. Chem. B 114(8), 2549-2564 (2010).

${ }^{29}$ H. S. Antila and E. Salonen, "Polarizable force fields," Biomolecular Simulations (Springer, 2013), pp. 215-241.

${ }^{30}$ C. Kramer, T. Bereau, A. Spinn, K. R. Liedl, P. Gedeck, and M. Meuwly, "Deriving static atomic multipoles from the electrostatic potential," J. Chem. Inf. Model. 53, 3410-3417 (2013).

${ }^{31} \mathrm{H}$. B. G. Casimir and D. Polder, "The influence of retardation on the London-van der Waals forces," Phys. Rev. 73(4), 360 (1948).

${ }^{32} \mathrm{X}$. Chu and A. Dalgarno, "Linear response time-dependent density functional theory for van der Waals coefficients," J. Chem. Phys. 121, 4083 (2004).

${ }^{33}$ A. Okabe, B. Boots, K. Sugihara, and S. N. Chiu, Spatial Tessellations: Concepts and Applications of Voronoi Diagrams, 2nd ed. (Wiley, 2009), Vol. 501.

${ }^{34}$ A. Ambrosetti, A. M. Reilly, R. DiStasio, Jr., and A. Tkatchenko, "Long range correlation energy from coupled atomic response functions," J. Chem. Phys. 140, 18A508 (2014).

${ }^{35}$ J. Applequist, J. R. Carl, and K.-K. Fung, "Atom dipole interaction model for molecular polarizability: Application to polyatomic molecules and determination of atom polarizabilities," J. Am. Chem. Soc. 94(9), 2952-2960 (1972)

${ }^{36}$ J. Applequist, "On the polarizability theory of optical rotation," J. Chem. Phys. 58(10), 4251-4259 (1973).

${ }^{37}$ A. Stone, The Theory of Intermolecular Forces, 2nd ed. (Oxford University Press, 2013).

${ }^{38}$ B. R. Brooks, C. L. Brooks, A. D. Mackerell, L. Nilsson, R. J. Petrella, B. Roux, Y. Won, G. Archontis, and C. Bartels, S. Boresch et al., "Charmm: The biomolecular simulation program," J. Comput. Chem. 30(10), 15451614 (2009).

${ }^{39}$ T. Bereau, C. Kramer, and M. Meuwly, "Leveraging symmetries of static atomic multipole electrostatics in molecular dynamics simulations," J. Chem. Theory Comput. 9(12), 5450-5459 (2013).

${ }^{40}$ T. Bereau, C. Kramer, F. W. Monnard, E. S. Nogueira, T. R. Ward, and M. Meuwly, "Scoring multipole electrostatics in condensed-phase atomistic simulations," J. Phys. Chem. B 117(18), 5460-5471 (2013).
${ }^{41}$ B. T. Thole, "Molecular polarizabilities calculated with a modified dipole interaction," Chem. Phys. 59(3), 341-350 (1981).

${ }^{42}$ P. Jurečka, J. Šponer, J. Černỳ, and P. Hobza, "Benchmark database of accurate (MP2 and CCSD (T) complete basis set limit) interaction energies of small model complexes, DNA base pairs, and amino acid pairs," Phys. Chem. Chem. Phys. 8(17), 1985-1993 (2006).

${ }^{43} \mathrm{LDA}(2.05 \mathrm{kcal} / \mathrm{mol})$, HSE $(2.38 \mathrm{kcal} / \mathrm{mol})$, and PBE $(2.72 \mathrm{kcal} / \mathrm{mol})$ are comparable, while B3LYP shows a $3.81 \mathrm{kcal} / \mathrm{mol}$ error. ${ }^{46}$

${ }^{44}$ R. S. Paton and J. M. Goodman, "Hydrogen bonding and $\pi$-stacking: How reliable are force fields? A critical evaluation of force field descriptions of nonbonded interactions," J. Chem. Inf. Model. 49(4), 944-955 (2009).

${ }^{45}$ A. P. Jones, J. Crain, V. P. Sokhan, T. W. Whitfield, and G. J. Martyna, "Quantum drude oscillator model of atoms and molecules: Many-body polarization and dispersion interactions for atomistic simulation," Phys. Rev. B 87(14), 144103 (2013).

${ }^{46}$ N. Marom, A. Tkatchenko, M. Rossi, V. V. Gobre, O. Hod, M. Scheffler, and L. Kronik, "Dispersion interactions with density-functional theory: Benchmarking semiempirical and interatomic pairwise corrected density functionals," J. Chem. Theory Comput. 7(12), 3944-3951 (2011).

${ }^{47}$ T. Bučko, S. Lebegue, J. Hafner, and J. G. Ángyán, "Tkatchenko-Scheffler van der Waals correction method with and without self-consistent screening applied to solids," Phys. Rev. B 87(6), 064110 (2013).

${ }^{48} \mathrm{Q}$. Wu and W. Yang, "Empirical correction to density functional theory for van der Waals interactions," J. Chem. Phys. 116(2), 515-524 (2002).

${ }^{49}$ J. Řezáč, P. Jurečka, K. E. Riley, J. Černỳ, H. Valdes, K. Pluháčková, K. Berka, T. Řezáč, and M. Pitoňák, J. Vondrášek et al., "Quantum chemical benchmark energy and geometry database for molecular clusters and complex molecular systems (www. begdb. com): A users manual and examples," Collect. Czech. Chem. C. 73(10), 1261-1270 (2008).

${ }^{50}$ L. Gráfová, M. Pitoňák, J. Řezáč, and P. Hobza, "Comparative study of selected wave function and density functional methods for noncovalent interaction energy calculations using the extended s22 data set," J. Chem. Theory Comput. 6(8), 2365-2376 (2010).

${ }^{51}$ J. Rezác, K. E. Riley, and P. Hobza, "S66: A well-balanced database of benchmark interaction energies relevant to biomolecular structures," J. Chem. Theory Comput. 7(8), 2427-2438 (2011).

${ }^{52}$ J. Řezáč and P. Hobza, "Advanced corrections of hydrogen bonding and dispersion for semiempirical quantum mechanical methods," J. Chem. Theory Comput. 8(1), 141-151 (2012).

${ }^{53}$ K. Berka, R. Laskowski, K. E. Riley, P. Hobza, and J. Vondrášek, "Representative amino acid side chain interactions in proteins: A comparison of highly accurate correlated $a b$ initio quantum chemical and empirical potential procedures," J. Chem. Theory Comput. 5(4), 982-992 (2009).

${ }^{54}$ J. Řezáč, K. E. Riley, and P. Hobza, "Benchmark calculations of noncovalent interactions of halogenated molecules," J. Chem. Theory Comput. 8(11), 4285-4292 (2012).

${ }^{55}$ B. Temelso, K. A. Archer, and G. C. Shields, "Benchmark structures and binding energies of small water clusters with anharmonicity corrections," J. Phys. Chem. A 115(43), 12034-12046 (2011).

${ }^{56}$ M. J. Gillan, D. Alfè, P. J. Bygrave, C. R. Taylor, and F. R. Manby, "Energy benchmarks for water clusters and ice structures from an embedded manybody expansion,” J. Chem. Phys. 139(11), 114101 (2013).

${ }^{57}$ B. Santra, A. Michaelides, and M. Scheffler, "On the accuracy of densityfunctional theory exchange-correlation functionals for $\mathrm{h}$ bonds in small water clusters: Benchmarks approaching the complete basis set limit," J. Chem. Phys. 127, 184104 (2007).

${ }^{58}$ B. Santra, A. Michaelides, M. Fuchs, A. Tkatchenko, C. Filippi, and M. Scheffler, "On the accuracy of density-functional theory exchangecorrelation functionals for $\mathrm{h}$ bonds in small water clusters. II. The water hexamer and van der Waals interactions," J. Chem. Phys. 129, 194111 (2008).

${ }^{59}$ A. Defusco, D. P. Schofield, and K. D. Jordan, "Comparison of models with distributed polarizable sites for describing water clusters," Mol. Phys. 105(19-22), 2681-2696 (2007).

${ }^{60}$ T. Sommerfeld and K. D. Jordan, "Quantum Drude oscillator model for describing the interaction of excess electrons with water clusters: An application to (h2o) 13," J. Phys. Chem. A 109(50), 11531-11538 (2005)

${ }^{61}$ T. Sommerfeld and K. D. Jordan, "Electron binding motifs of (h2o) nclusters," J. Am. Chem. Soc. 128(17), 5828-5833 (2006).

${ }^{62} \mathrm{P}$. Ren and J. W. Ponder, "Polarizable atomic multipole water model for molecular mechanics simulation," J. Phys. Chem. B 107(24), 5933-5947 (2003) 
${ }^{63}$ D. A. Bardwell, C. S. Adjiman, Y. A. Arnautova, E. Bartashevich, S. X. M. Boerrigter, D. E. Braun, A. J. Cruz-Cabeza, G. M. Day, and R. G. Della Valle, G. R. Desiraju et al., "Towards crystal structure prediction of complex organic compounds-a report on the fifth blind test," Acta Crystallogr. B 67(6), 535-551 (2011).

${ }^{64}$ Note that the current implementation does not allow to relax internal coordinates.

${ }^{65}$ E. Tapavicza, I.-C. Lin, O. A. von Lilienfeld, I. Tavernelli, M. D. CoutinhoNeto, and U. Rothlisberger, "Weakly bonded complexes of aliphatic and aromatic carbon compounds described with dispersion corrected density functional theory," J. Chem. Theory Comput. 3(5), 1673-1679 (2007).

${ }^{66}$ W. B. Schweizer and J. D. Dunitz, "Quantum mechanical calculations for benzene dimer energies: Present problems and future challenges," J. Chem. Theory Comput. 2(2), 288-291 (2006).

${ }^{67}$ I.-C. Lin, O. A. von Lilienfeld, M. D. Coutinho-Neto, I. Tavernelli, and U. Rothlisberger, "Predicting noncovalent interactions between aromatic biomolecules with London-dispersion-corrected DFT," J. Phys. Chem. B 111(51), 14346-14354 (2007)

${ }^{68}$ A. Benali, L. Shulenburger, N. Romero, A. J. Kim, and O. A. von Lilienfeld, "Application of diffusion Monte Carlo to materials dominated by van der Waals interactions," J. Chem. Theory Comput. (published online, 2014).

${ }^{69}$ W. Humphrey, A. Dalke, and K. Schulten, "VMD: Visual molecular dynamics," J. Mol. Graph. Model. 14(1), 33-38 (1996).
${ }^{70} \mathrm{~S}$. Grimme, "Supramolecular binding thermodynamics by dispersioncorrected density functional theory," Chem. Eur. J. 18(32), 9955-9964 (2012).

${ }^{71}$ T. Risthaus and S. Grimme, "Benchmarking of London dispersionaccounting density functional theory methods on very large molecular complexes," J. Chem. Theory Comput. 9(3), 1580-1591 (2013).

${ }^{72}$ A. Ambrosetti, D. Alfè, R. DiStasio, and A. Tkatchenko, "Hard numbers for large molecules: Towards exact energetics for supramolecular systems," J. Phys. Chem. Lett. 5, 849-855 (2014).

${ }^{73}$ Y. Zhao and D. G. Truhlar, "The m06 suite of density functionals for main group thermochemistry, thermochemical kinetics, noncovalent interactions, excited states, and transition elements: Two new functionals and systematic testing of four m06-class functionals and 12 other functionals," Theor. Chem. Acc. 120(1-3), 215-241 (2008).

${ }^{74}$ J. D. Weeks, D. Chandler, and H. C. Andersen, "Role of repulsive forces in determining the equilibrium structure of simple liquids," J. Phys. Chem. 54, 5237 (1971).

${ }^{75}$ T. A. Halgren, "The representation of van der Waals (vdW) interactions in molecular mechanics force fields: Potential form, combination rules, and vdW parameters," J. Am. Chem. Soc. 114(20), 7827-7843 (1992).

${ }^{76} \mathrm{~T}$. W. Whitfield and G. J. Martyna, "A unified formalism for many-body polarization and dispersion: The quantum Drude model applied to fluid xenon," Chem. Phys. Lett. 424(4), 409-413 (2006). 